\title{
Refined surgical techniques to improve the patency of cryopreserved iliac artery homografts for middle hepatic vein reconstruction during living donor liver transplantation
}

\author{
Gil-Chun Park, Shin Hwang, Dong-Hwan Jung, Tae-Yong Ha, Gi-Won Song, Chul-Soo Ahn, Deok-Bog Moon, \\ Ki-Hun Kim, Young-In Yoon, Hwui-Dong Cho, Jin-Uk Choi, Sung-Gyu Lee
}

Division of Liver Transplantation and Hepatobiliary Surgery, Department of Surgery, Asan Medical Center, University of Ulsan College of Medicine, Seoul, Korea

\begin{abstract}
Purpose: A cryopreserved iliac artery homograft (IAH) has not been considered suitable for middle hepatic vein (MHV) reconstruction during living donor liver transplantation (LDLT), primarily due to the low patency from its small diameter. We revised our surgical techniques for MHV reconstruction using an IAH to improve its patency.

Methods: This study analyzed the causes of early conduit occlusion and developed revised techniques to address this that had clinical application.

Results: The potential risk factors for early conduit occlusion were the small IAH size, small graft in the segment $\mathrm{V}$ vein (V5) and segment VIII vein (V8) opening, and small recipient MHV-left hepatic vein stump. These factors were reflected to our revised surgical methods which included endarterectomy of the atherosclerotic plaque, unification of the internal and external iliac artery branches for large V5, and branch-patch arterioplasty for large V8. IAH endarterectomy, branch unification technique, and branch-patch arterioplasty were applied to 8,5 , and 5 patients, respectively and resulted in 1 -month occlusion rates of $37.5 \%, 20.0 \%$, and $40.0 \%$, respectively. The overall patency rates of the IAH-MHV conduits in our 18 patients were $66.7 \%$ at 1 month, $38.9 \%$ at 3 months, and $33.3 \%$ at 1 year.

Conclusion: Our refined MHV reconstruction using an IAH improved short-term MHV conduit patency, but did not effectively prevent early conduit occlusion, particularly with a small- or medium-sized IAH. Individualized reconstruction designs during LDLT operation are needed when an IAH is used for a modified right liver graft.

[Ann Surg Treat Res 2020;99(5):294-304]
\end{abstract}

Key Words: Arterioplasty, Endarterectomy, Hepatic venous congestion, Iliac vein graft

\section{INTRODUCTION}

Middle hepatic vein (MHV) reconstruction with vessel graft interposition is one of the standard procedures for living donor liver transplantation (LDLT) using a modified right lobe graft. Various interposition conduit materials have been used for this procedure, including homologous and autologous vessels and prosthetic vascular grafts [1-7]. Since the supply of vascular homografts is very limited in Korea, we have used prosthetic vascular grafts including ringed polytetrafluoroethylene and collagen-impregnated woven double velour polyester grafts (Hemashield Platinum, Maquet, Rastatt, Germany) in
Received April 14, 2020, Revised July 16, 2020, Accepted July 30, 2020

\section{Corresponding Author: Shin Hwang}

Division of Liver Transplantation and Hepatobiliary Surgery, Department of Surgery, Asan Medical Center, University of Ulsan College of Medicine, 88 Olympic-ro 43-gil, Songpa-gu, Seoul 05505, Korea

Tel: +82-2-3010-3930, Fax: +82-2-3010-6701

E-mail: shwang@amc.seoul.kr

ORCID: https://orcid.org/0000-0002-9045-2531

\section{Copyright (c) 2020, the Korean Surgical Society}

(c) Annals of Surgical Treatment and Research is an Open Access Journal. All articles are distributed under the terms of the Creative Commons Attribution NonCommercial License (http://creativecommons.org/licenses/by-nc/4.0/) which permits unrestricted non-commercial use, distribution, and reproduction in any medium, provided the original work is properly cited. 
combination with small-artery patches [6,7]. The short- and long-term patency rates of these prosthetic graft conduits have proved to be comparable to those of cryopreserved iliac vein and aorta homografts [5-7]. Notably however, some prosthetic graftassociated complications such as accidental gastric penetration by the graft have sporadically occurred [8-11]. Furthermore, the majority of prosthetic graft conduits remain nonfunctioning foreign bodies after thrombotic luminal occlusion, leading to a potential risk of other complications that are directly associated with prosthetic grafts. It is thus reasonable to avoid the use of prosthetic materials for grafting whenever possible.

Next to large-sized homografts such as a cryopreserved iliac vein and aorta, a cryopreserved iliac artery homograft (IAH) appears to be a useful material for MHV reconstruction. However, we previously reported that the short-term patency rates of the IAH conduits were too low to reliably use them for MHV reconstruction because of their relatively small diameter $[2,4,6]$. However, since the supply of the relatively large-sized IAHs is very limited, we wished to improve the patency rates of the medium-sized IAHs through technical innovation.

We herein present our refined surgical techniques that were applied to MHV reconstruction using IAHs and our analysis of their effectiveness, with a focus on the short-term patency outcomes.

\section{METHODS}

\section{Study design and patient selection}

This study consisted of 2 parts. The first part of this study was an analysis of the underlying causes of early luminal occlusion after MHV reconstruction using a cryopreserved IAH. The second part involved the development of refined surgical techniques that could be flexibly applied to the diverse matching status of an IAH and graft MHV branch openings.

In the first part of this study, we selected cases showed an early occlusion of their IAH conduits during a 6-year period from January 2010 to December 2015. Early occlusion was defined as loss of conduit blood flow within 1 month of LDLT operation. The investigation parameters were the IAH diameters, branching pattern of the external and internal IAHs, number and size of the graft MHV branch openings, and anastomosis types of an IAH to the recipient inferior vena cava (IVC). The sequences of IAH conduit occlusion on serial dynamic CT scans were analyzed to determine the mechanisms of early occlusion.

In the second part of this study, we retrospectively reviewed our LDLT cases involving an IAH over the 3-year period from January 2016 to December 2018. The investigation parameters were the types of IAH anastomosis to the graft MHV branch openings and to the recipient IVC. The patency of the IAH conduits was analyzed using the serial dynamic CT scanning after the LDLT operation.

The inclusion criteria for patient selection were adult patients who had undergone an LDLT operation with a modified right liver graft and MHV reconstruction using an IAH conduit. The exclusion criteria were a lack of precise information on the IAHs and reconstruction techniques in the operation records, a single reconstruction of the segment VIII vein (V8) or segment $\mathrm{V}$ vein (V5) orifice, a lack of regular dynamic CT follow-ups (usually due to renal dysfunction), and patient death or hepatocellular carcinoma recurrence within the first 2 years after LDLT.

Our current study protocol was approved by the Institutional Review Board of our institution (No. 2019-0524), which waived the requirement for informed consent due to the retrospective nature of this study. This study was performed in accordance with the ethical guidelines of the World Medical Association Declaration of Helsinki 2013.

\section{Evaluation of IAH-interposed MHV conduit patency and indications for interventional stenting}

In accordance with our institutional LDLT management protocols, posttransplant dynamic CT scans were taken weekly when the patients were in the hospital and at 1, 3, 6, and 12 months after the liver transplantation operation. Thereafter, a follow-up abdomen-pelvis CT was conducted annually for 5 years and biannually after 5 years [7].

Interventional stenting of the thrombosed MHV conduit was indicated if a significant MHV occlusion-related perfusion abnormality occurred in the graft liver [12-15]. We assessed the amount and nature of $\mathrm{CT}$ attenuation changes. The definitions of $\mathrm{CT}$ attenuation abnormalities, such as hypoattenuation and hyperattenuation, have been described previously $[14,15]$.

We regarded MHV stenting as occlusion of the MHV conduit graft, although the MHV conduit patency was restored after this intervention.

\section{Homograft cryopreservation and defrost}

All homografts used in this study were obtained from Korea Public Tissue Bank (Seoul, Korea) and the institutional tissue bank of Asan Medical Center. All human tissues stored at these tissue banks were donated after the informed consent of donors' family members. All procedures for vascular tissue procurement and processing were in compliance with Korean legislation (Law 5858/1999 and Law 11976/2013) and conformed to the ethical and safety concerns for therapeutic use.

The IAH was washed with saline solution and stored at $4^{\circ} \mathrm{C}$. For cryopreservation, washed arterial allografts were immersed in cryopreservation solution, consisting of $90 \%$ culture medium (RPMI 1640) and 10\% dimethyl sulfoxide (DMSO) in a cryostorage bag at $20^{\circ} \mathrm{C}$. The bag was sealed in a laminar flow cabinet. Programmed cryopreservation was performed in a controlled rate freezing system. The protocol consisted of 
a slow, programmed cooling at a mean rate of $1^{\circ} \mathrm{C}$ /minute, to $-70^{\circ} \mathrm{C}$. The bag was immediately transferred to the gas phase of a liquid nitrogen compartment, followed by rapid cooling to $-196^{\circ} \mathrm{C}[16,17]$.

The defrost protocol was a 2-stage rewarming process, consisting of slow warming by transferring the bag from the nitrogen gas phase to room temperature $\left(20^{\circ} \mathrm{C}\right)$ over $10-30$ minutes, followed by rapid warming by immersing the bag in a water bath at $40^{\circ} \mathrm{C}$ until the contents were completely defrosted. The cryoprotectant liquid was gradually removed in four 3-minute steps by immersion in tapering concentrations of DMSO $(10 \%, 5 \%, 0 \%$, and $0 \%)$ at $4^{\circ} \mathrm{C}[16,17]$.

\section{Statistical analysis}

All numerical data were presented as mean values with standard deviations. Conduit patency rates were determined using the Kaplan-Meier method. Statistical analyses were performed using IBM SPSS Statistics ver. 22 (IBM Corp., Armonk, NY, USA).

\section{RESULTS}

\section{Mechanisms of early occlusion of IAH conduits}

Fifteen LDLT recipients (53.6\%) out of 28 patients in the assigned study period showed early occlusion of their IAH conduit within the first month after their LDLT operation. Their mean age was $54.1 \pm 8.7$ years. Male patients were 11
(73.3\%). HBV infection-associated liver cirrhosis was present in $11(73.3 \%)$. The model for end-stage liver disease score was 16.7 \pm 6.3 . ABO blood-incompatible LDLT was in 2 (13.3\%). The graftrecipient weight ratio was $1.13 \pm 0.21$.

The occlusion patterns in the IAH conduits during the first month were classified as concurrent occlusion of the V5 and V8 (type $1, \mathrm{n}=9$ ) or as early occlusion of the V5 and delayed occlusion of the V8 (type $2, \mathrm{n}=6$ ). The mean outer diameter of IAHs at the proximal common iliac artery level was $9.6 \pm$ $1.9 \mathrm{~mm}$ in 15 patients with early occlusion within 1 month and $10.6 \pm 1.4 \mathrm{~mm}$ in 13 patients without early occlusion $(\mathrm{P}=0.11)$.

Among the 9 patients with type 1 occlusion, the outer diameter of the common iliac artery was less than $10 \mathrm{~mm}$ (internal diameter $<7 \mathrm{~mm}$, regarded as a small-sized IAH) in 6 cases (66.7\%); the size of V5 or V8 opening at the graft cut surface was less than $6 \mathrm{~mm}$ in 7 cases (77.8\%); and a smallsized MHV-left hepatic vein (LHV) trunk stump $(<2 \mathrm{~cm}$ in the transverse diameter) was evident at the recipient IVC in 4 cases (44.4\%). Of the 9 type 1 occlusion cases, 5 patients (55.6\%) underwent percutaneous MHV stenting through the internal jugular vein route and the MHV flow was restored in 3 of these patients (33.3\%).

In the 6 patients with type 2 occlusion, the outer diameter of the common iliac artery was less than $10 \mathrm{~mm}$ in $2(33.3 \%)$; the size of the V5 or V8 opening was less than $6 \mathrm{~mm}$ in $3(50.0 \%)$; and a small-sized MHV-LHV trunk at the recipient IVC was detected in 1 case (16.7\%). Of these type 2 cases, 5 patients
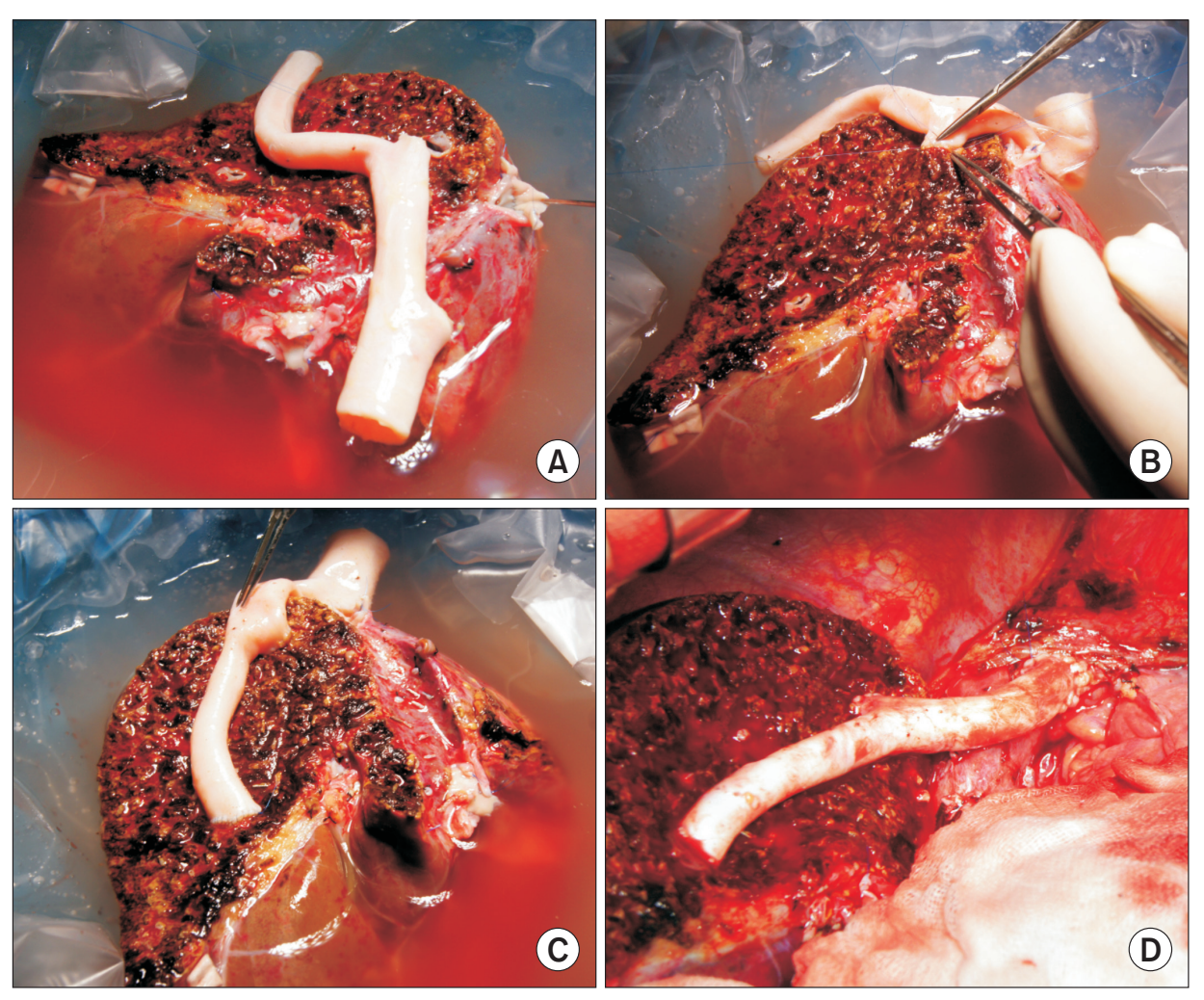

Fig. 1. Intraoperative photographs showing a conventional technique for middle hepatic vein reconstruction using a large-sized cryopreserved iliac artery homograft (IAH). No arterioplasty of the IAH was applied. (A) A largesized IAH was prepared. (B) The internal iliac artery branch was anastomosed to the graft segment VIII vein (V8) opening. (C) The external iliac artery branch was anastomosed to the graft $\mathrm{V} 5$ opening. (D) The IAH conduit was anastomosed to the middleleft hepatic vein stump of the recipient inferior vena cava. The conduit was patent for more than 1 year after transplantation. 

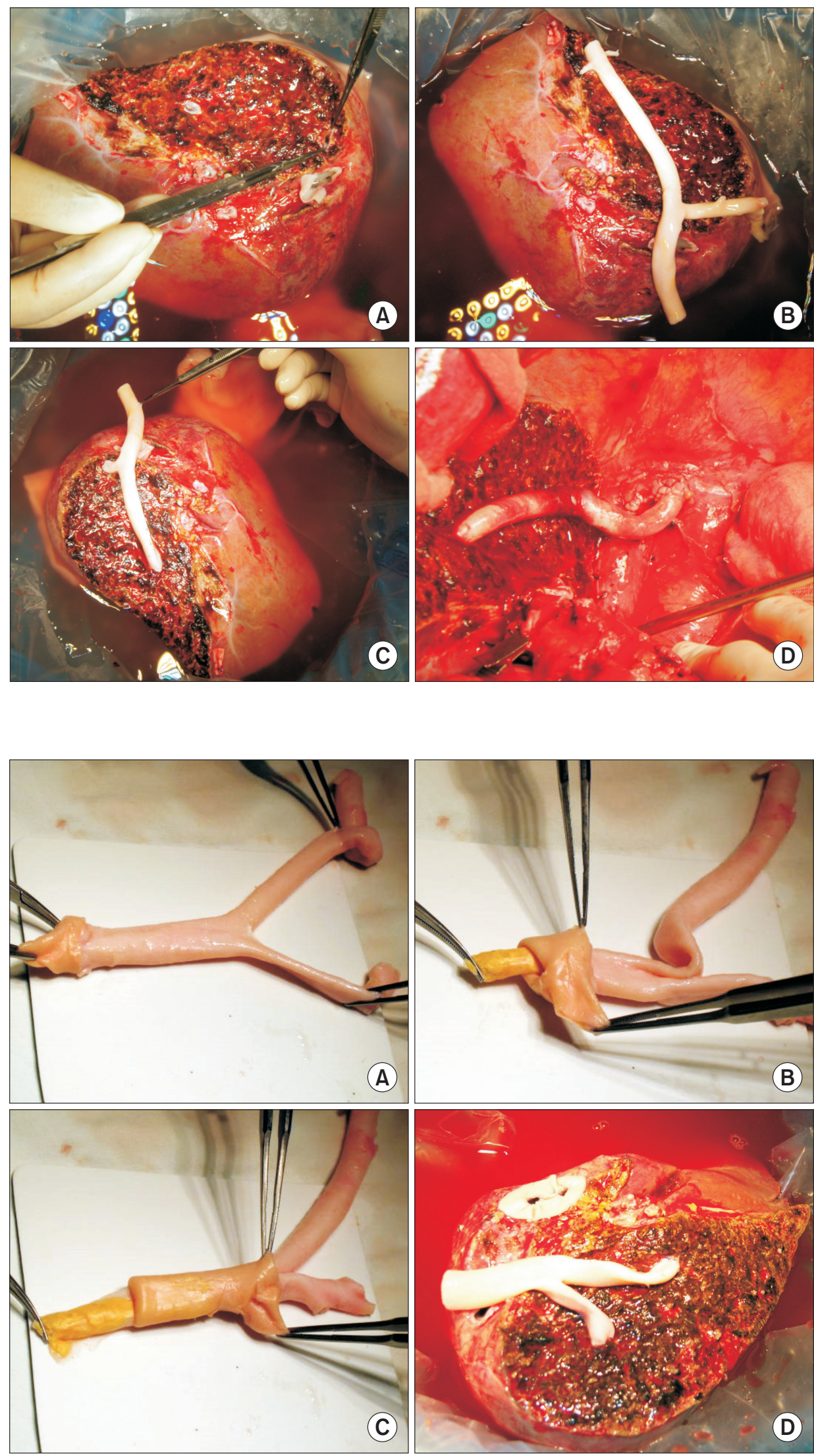

Fig. 2. Intraoperative photographs showing a suboptimal middle hepatic vein reconstruction technique using a small-sized cryopreserved iliac artery homograft (IAH). (A) A small-sized IAH was prepared. (B) The internal iliac artery branch was anastomosed to the graft segment VIII vein (V8) opening. (C) The external iliac artery branch was anastomosed to the graft $\mathrm{V} 5$ opening. (D) The recipient middle hepatic vein stump was closed for size matching and the IAH conduit was anastomosed only to the left hepatic vein stump of the recipient inferior vena cava. The conduit was occluded within 1 week after transplantation and transjugular stenting was therefore performed.
Fig. 3. Intraoperative photographs showing a successful ex situ endarterectomy procedure. (A) The outer contour of the cryopreserved iliac artery homograft (IAH) was examined. (B) The atheroma was gently pulled out through eversion of the adventitia. (C) The luminal surface of the adventitia was shiny, indicating a very smooth surface. (D) The endarterectomized $\mathrm{IAH}$ conduit was used for reconstruction of the graft middle hepatic vein branches. 
Table 1. Clinical profiles of the living donor liver transplantation recipients who underwent middle hepatic vein reconstruction using cryopreserved iliac artery homografts

\begin{tabular}{lccc}
\hline \multicolumn{1}{c}{ Variable } & Endarterectomy group & Branch unification group & Branch-patch arterioplasty group \\
\hline Patient & 8 & 5 & 5 \\
Recipient age $(y r)$ & $53.1 \pm 7.6$ & $51.7 \pm 8.2$ & $55.1 \pm 6.9$ \\
Male:female & $6: 2$ & $3: 2$ & $4: 1$ \\
Primary liver disease & & $3(60.0)$ & $3(60.0)$ \\
$\quad$ HBV-associated liver cirrhosis & $5(62.5)$ & $2(40.0)$ & $2(40.0)$ \\
$\quad$ Other disease & $3(37.5)$ & $2(40.0)$ & $3(60.0)$ \\
Concurrent hepatocellular carcinoma & $4(50.0)$ & $12.9 \pm 6.1$ & $13.8 \pm 71.0$ \\
Model for end-stage liver disease score & $14.7 \pm 3.4$ & $0(0)$ & $1(20.0)$ \\
ABO blood group-incompatible transplantation & $1(12.5)$ & $1.12 \pm 0.19$ & $1.07 \pm 0.13$ \\
Graft-recipient weight ratio & $1.09 \pm 0.18$ & $9.1 \pm 1.9$ & $9.0 \pm 2.1$ \\
Size of iliac artery homograft (mm) & $9.4 \pm 1.8$ & $3(20.0)$ & $2(40.0)$ \\
Early conduit occlusion within 1 month & $3(37.5)$ & $3(60.0)$ & $4(80.0)$ \\
Cumulative conduit occlusion at 1 year & $5(62.5)$ & & \\
\hline
\end{tabular}

Values are presented as number only, mean \pm standard deviation, or number $(\%)$.

${ }^{\text {a) }}$ Outer diameter of the proximal common iliac artery.

underwent MHV stenting as the site of V8 anastomosis was suspected of being stenotic. The MHV flow was regained in all 5 patients $(100 \%)$.

These results indicated that the potential risk factors for early conduit occlusion among our study cases included the small size of the IAH (8 [53.3\%]), a small graft V5 or V8 opening (10 [66.7\%]), and small MHV-LHV stump (5 [33.3\%]). Early occlusion developed in 7 of 8 patients (87.5\%) in which a small-sized IAH conduit was anastomosed to a small $\mathrm{V} 8$ orifice.

\section{Technical modification for MHV reconstruction using IAHs and the resulting patency outcomes}

The conventional techniques for MHV reconstruction using cryopreserved IAHs without arterioplasty are presented in Figs. 1 and 2 . The external and internal iliac artery branches were anastomosed to the graft V5 and V8 openings, respectively.

Our revised surgical techniques to address the 3 abovementioned risk factors for early occlusion were classified into 3 types as follows; endarterectomy of the atherosclerotic plaque, unification of the internal and external iliac artery branches for a large V5, and branch-patch arterioplasty for a large V8.

Endarterectomy of the IAH (Fig. 3) was conducted in 8 of our study patients (Table 1). In 5 of these IAHs showing severe atherosclerosis, the atherosclerotic plaque was successfully peeled off all along the common and external iliac arteries. Such enlarged IAH conduits consisting of only adventitia were used for MHV reconstruction in these 5 cases as is done with iliac vein homografts. None of these 5 patients experienced early MHV conduit occlusion requiring stenting. In contrast, the atherosclerotic plaque was successfully peeled off at the common iliac vein portion, but not at the external iliac artery branch in other 3 cases of an IAH with moderate atherosclerosis
(Fig. 4). Forceful extraction of the atheroma resulted in a friable conduit that was consisted of only a paper-thin adventitia. Although the same abovementioned reconstruction technique was applied, all 3 patients in this case experienced early conduit occlusion and MHV flow was regained after stenting.

Unification of the internal and external iliac artery branches (Fig. 5) was applied to the right liver grafts with a relatively large V5 opening compared with the size of the external iliac artery branch. This technique was applied to 5 patients (Table 1). Unification sutures of the internal and external iliac artery branches enlarged the luminal diameter to a size comparable to that of the common iliac artery. One patient experienced a type 1 occlusion early after the LDLT operation, which required stenting. The remaining 4 patients did not undergo stenting for early MHV occlusion. Our modified unification technique for single V5 or V8 reconstruction is also presented in Fig. 6.

Branch-patch arterioplasty was applied to the right liver grafts with a relatively large V8 opening compared with the size of the internal iliac artery branch (Figs. 7, 8). Since direct anastomosis of the internal iliac artery branch to the V8 opening often resulted in a type 2 occlusion, a bulged portion was made at the V8 anastomosis site. This enlarged dome-roof like portion prevented anastomotic stenosis, which hemodynamically facilitated the MHV outflow drainage from the V5 and V8. This technique was applied to 5 of our current study patients (Table 1). Two of these cases in which relatively small-sized IAHs were used experienced a type 1 and 2 occlusion respectively and stenting was therefore carried out. The remaining 3 patients did not undergo stenting for early MHV occlusion.

Six patients out of our total study cohort of 18 cases experienced early MHV conduit occlusion within one month of the LDLT operation. The overall patency rates of the IAH- 

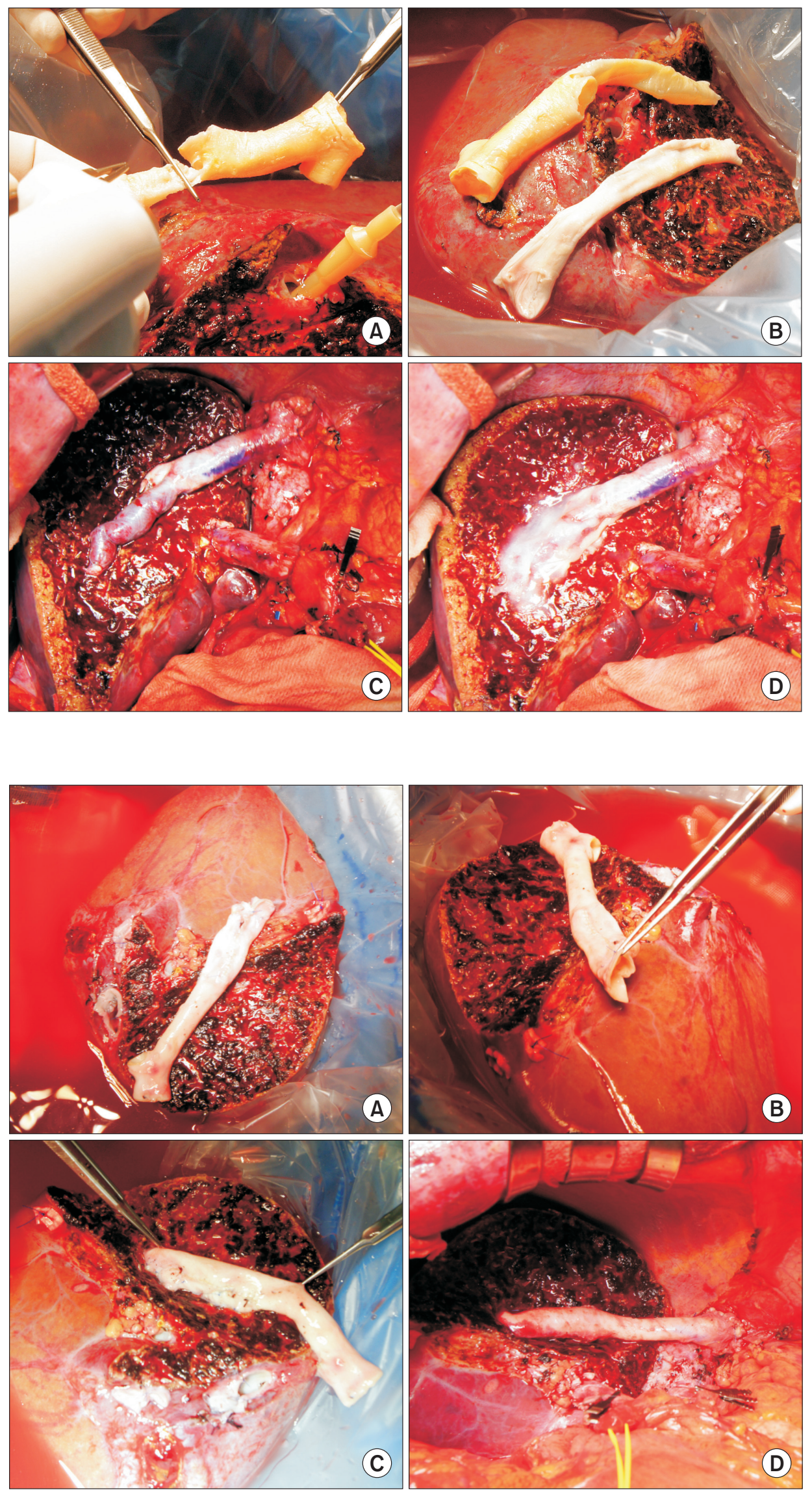

Fig. 4. Intraoperative photographs showing a suboptimal ex situ endarterectomy procedure. (A) The atheroma was forcefully pulled out because it was not easily detached from the adventitia. (B) The rough surface of the atheroma plaque reflected the counterpart surface status of the adventitial lumen. (C) The endarterectomized iliac artery homograft $(\mathrm{IAH})$ conduit was used for reconstruction of the graft middle hepatic vein branches. The external iliac artery portion appeared to be very thin. (D) The paper-thin portion of the $\mathrm{IAH}$ conduit was reinforced by spraying fibrin glue. The graft segment $V$ vein (V5) was occluded within 1 week after transplantation, but stenting was not performed because the $\mathrm{V} 8$ flow was preserved.
Fig. 5. Intraoperative photographs showing the branch unification technique. (A) The internal and external iliac artery branches and their crotch portion were incised and their anterior and posterior walls were sutured to enlarge the luminal diameter. (B) The unification sutures were designed to exactly match to the graft middle hepatic vein openings. (C) The graft segment $\mathrm{V}$ vein $(\mathrm{V} 5)$ was anastomosed in the end-to-end mode and the graft $\checkmark 8$ was reconstructed in the endto-side mode. (D) The conduit was used for reconstruction of the graft middle hepatic vein branches. 

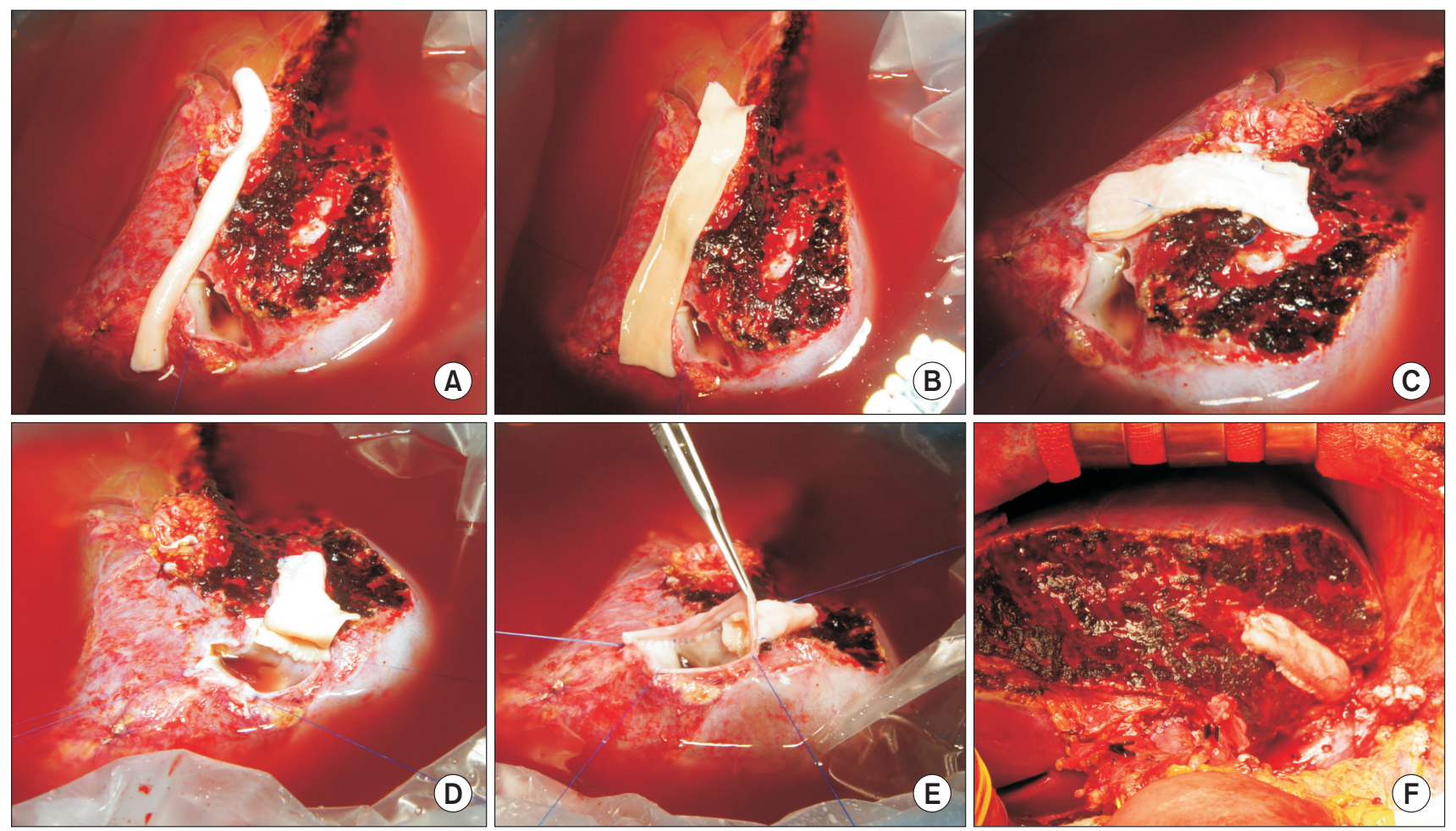

Fig. 6. Intraoperative photographs showing a modified branch unification technique for a single segment $\mathrm{V}$ vein (V5) or V8 reconstruction. (A) A small-sized external iliac artery-femoral artery homograft was prepared. (B) The artery was longitudinally incised. (C) Unification sutures converted the long patch into a large-sized tube. (D) The enlarged tube conduit was used for connection of the V8 and right hepatic vein orifice. (E) The right hepatic vein orifice was enlarged using a caudal incision and patch plasty. (F) The unified orifice of the right graft outflow vein was anastomosed to the recipient right hepatic vein stump.
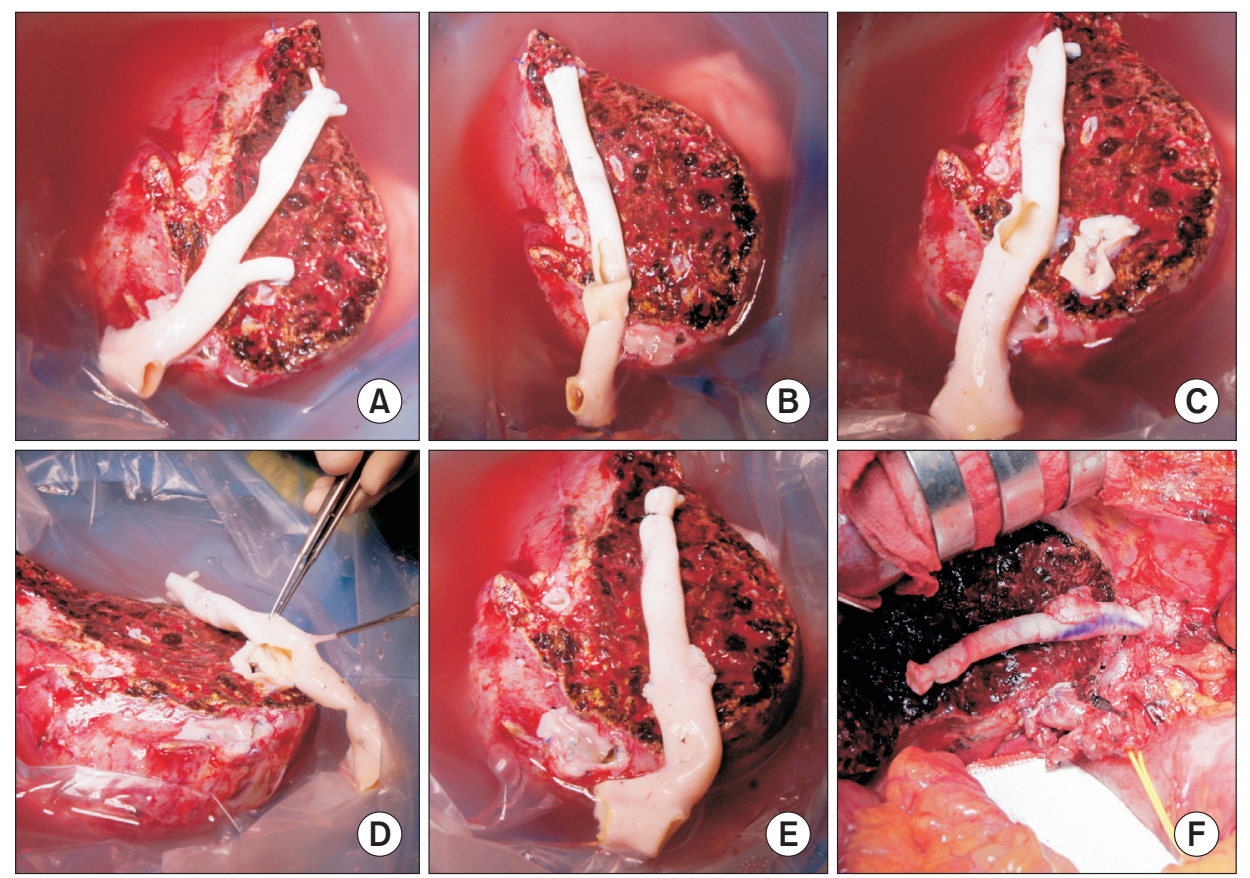

Fig. 7. Intraoperative photographs showing the procedures for branch-patch arterioplasty. (A) A large-sized iliac artery homograft (IAH) was prepared. (B) The internal iliac artery branch and its crotch portion were incised longitudinally and some of the external iliac artery wall was excised. (C) The dorsal side of the internal iliac artery patch and the corresponding external iliac artery wall was anastomosed to form a dome-like roof. An arterial patch was attached to the graft segment VIII vein (V8) opening. (D) The dome-like orifice was attached to the V8 opening that was enlarged by using patch plasty. (E) The graft $\checkmark 5$ was anastomosed in the endto-side mode. (F) The IAH conduit was used for reconstruction of the graft middle hepatic vein branches. 

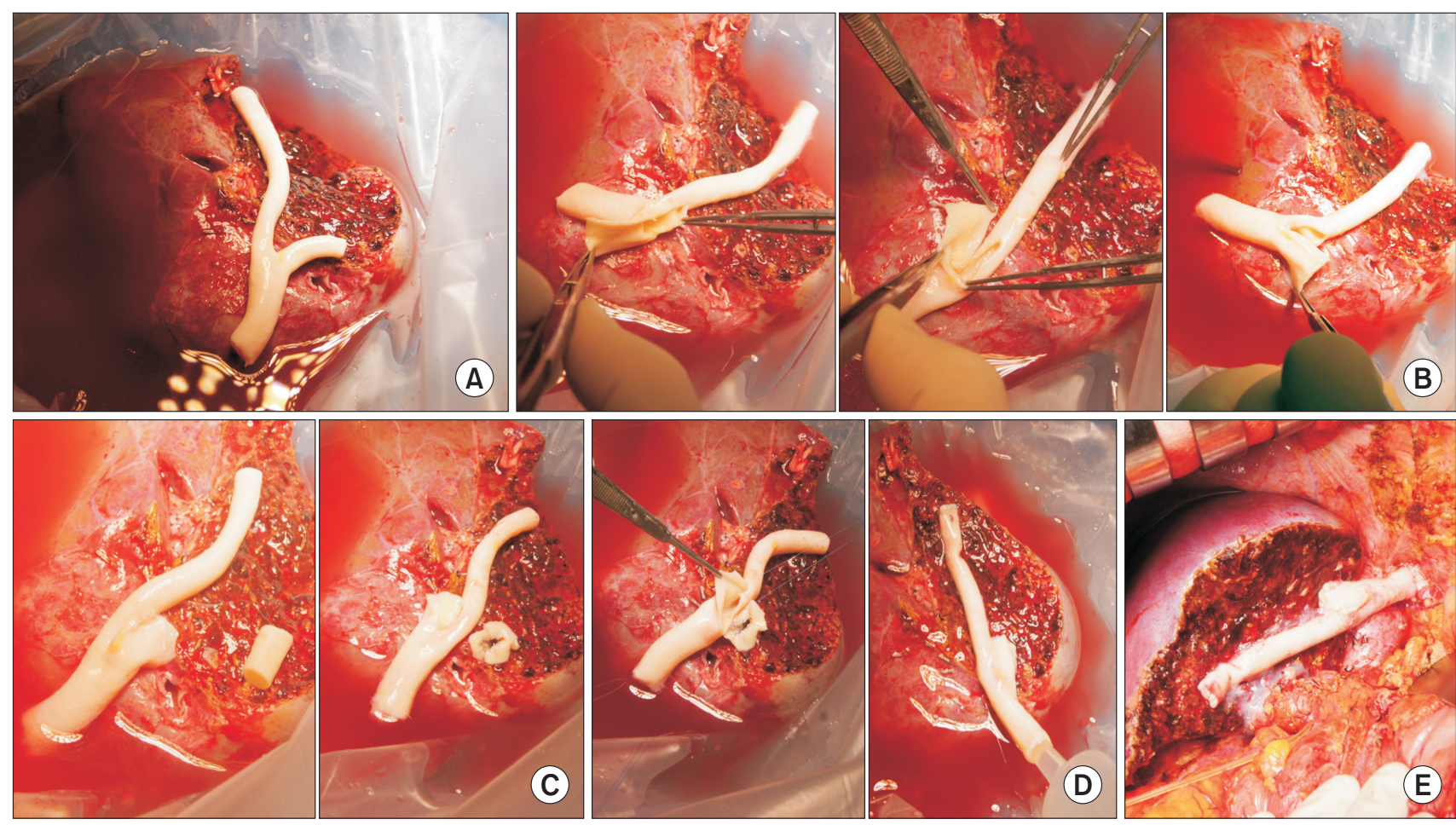

Fig. 8. Intraoperative photographs showing the procedures for branch-patch arterioplasty. (A) A medium-sized iliac artery homograft (IAH) was prepared. (B) The internal iliac artery branch was incised longitudinally and some of the external iliac artery wall was incised. The dorsal side of the internal iliac artery patch and the external iliac artery wall was anastomosed to form a dome-like roof and enlarged orifice. (C) An arterial patch was attached to the graft segment VIII vein (V8) opening. (D) The graft V8 and V5 openings were anastomosed to the IAH conduit. (E) The IAH conduit was used for reconstruction of the graft middle hepatic vein branches.

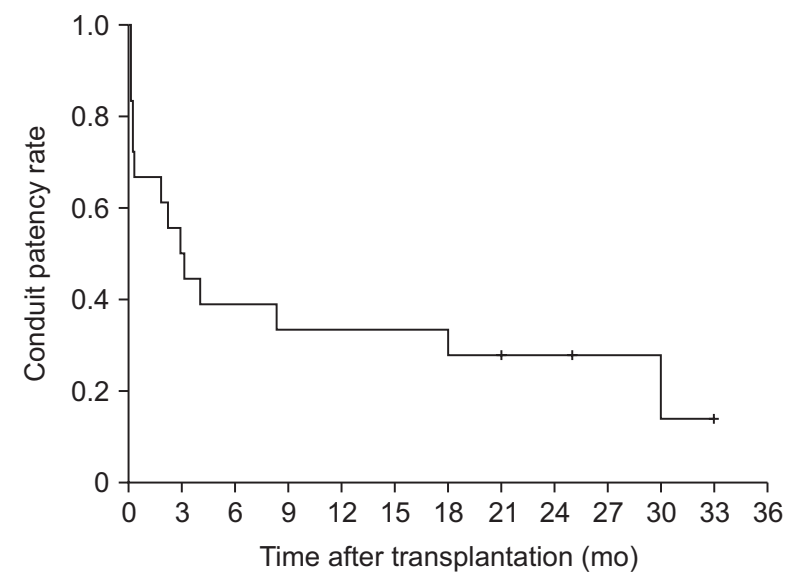

Fig. 9. Luminal patency curve for the middle hepatic vein reconstruction using cryopreserved iliac artery homografts.

MHV conduits in this series were $66.7 \%$ at 1 month, $38.9 \%$ at 3 months, 33.3\% at 1 year, and $27.9 \%$ at 2 years (Fig. 9).

\section{DISCUSSION}

MHV reconstruction for LDLT using a modified right liver graft has resulted in new demands for vascular homografts, and the increase in LDLT numbers had led to a relative shortage in their supply. Every available vessel material has been used for MHV reconstruction in many institutions, including various vascular homografts, prosthetic vascular grafts and autologous veins [2-7].

Prosthetic vascular grafts have the definite advantage of an unlimited supply but also have some critical disadvantages relative to hollow viscus penetration and the higher rate of complications associated with a foreign body [5,9-11]. Hence, it is clinically appropriate to avoid the use of prosthetic vascular grafts if possible on patient safety grounds. However, the advantages of prosthetic vascular grafts are at present far greater that the disadvantages of not conducting MHV reconstruction, and these artificial materials are thus currently regarded a "necessary evil" [5,7].

For our current study, we searched for new cryopreserved vascular homografts, which were stored in the tissue bank of our institution. We previously reported on the usability of cryopreserved aorta homografts that showed the highest patency rates [6]. However, the supply of aorta homografts is very limited, and almost all have been already used for MHV reconstruction. The other source of cryopreserved vascular homografts in our tissue bank was IAHs. We have occasionally 
used IAHs for MHV reconstruction, but their short-term patency rates have been consistently found to be too low for their continued clinical application $[2,4,6]$. As a result, many small- and medium-sized IAHs have remained unused in our tissue bank. The primary purpose of our present study was to evaluate the feasibility of using these IAHs as a MHV conduit.

Part 1 of our current study revealed that the potential risk factors for early occlusion of the IAH conduits were the small size of the graft V5 and V8 opening, small size of the IAH, and small MHV-LHV stump. Of these, the small size of the IAHs is the only factor that transplant surgeons can alter. Since we had already used the large-sized IAHs, only medium- or small-sized IAHs were available in our repository. A large-sized IAH can be used in a similar manner to an iliac vein homograft, but a medium-sized IAH cannot be used like in that manner because it does not have adequate patency rates for this purpose. Notably, it has been shown in a prior study that the short-term patency rate of the medium-sized IAHs is actually much lower than that of the prosthetic vascular grafts [6]. We therefore focused on how to improve the short-term patency rate of medium-sized IAHs in our current investigation.

We have previously presented the usability of ex situ endarterectomy of an IAH with severe atherosclerosis [4]. In practice however, removal of the atherosclerotic plaques during a carotid endarterectomy is not simple because the plaques are firmly attached to the adventitia layer. In contrast, atherosclerotic plaques are easily peeled off at the back table because the freezing and melting processes for a cryopreserved IAH loosen their attachment to the adventitia. Once these plaques are gently removed, the luminal surface of the adventitia becomes sufficiently smooth. This condition makes the endarterectomized IAH conduit less thrombogenic although there is no real endothelial lining. The endarterectomized IAH is soft and large-sized, thus its technical handling is comparable to that of iliac vein homografts [4].

It must be noted however that an ex situ endarterectomy is not always successful if the grade of atherosclerosis is not severe. If the atherosclerotic plaque is not smoothly detached from the adventitia layer, the luminal surface becomes rough and irregular and thus highly thrombogenic. At the external iliac artery in particular, forceful extraction of the intimal layer results in a friable conduit comprising a paper-thin adventitia, which is also highly thrombogenic. The lesson from our failure cases is that an endarterectomy should not be attempted if the atherosclerosis is not severe.

Unification arterioplasty of the internal and external iliac artery branches was found to be effective in generating a single long large-sized conduit without sacrifice of the conduit length, which is suitable for a large V5 opening. However, this is possible only when a long internal iliac artery branch is attached.
Branch-patch arterioplasty is a unique technique for preventing anastomotic stenosis at the $\mathrm{V} 8$ reconstruction which is a common cause of type 1 or type 2 occlusion of IAH conduits. The bulged dome roof-shaped portion facilitates a hemodynamic-compliant passage of the V5 outflow and pullingout of the V8 outflow. However, even with this technique, early occlusion developed in 2 of 5 patients in whom small-sized IAHs were used.

The aforementioned 3 types of technical innovation resulted in improved short-term patency rates of $66.7 \%$ at 1 month and $33.3 \%$ at 1 year in our 18 LDLT patients. These results were better than those we reported previously, which were $65.3 \%$ patency at 1 month, 35.2\% patency at 6 months, and 20.2\% at 1 year [5]. These results support that the patency of smalland medium-sized IAHs with our modified reconstruction techniques was comparable to that of large-sized IAHs with conventional reconstruction technique. However, 1/3 of the IAH conduits in our present study still became occluded within 1 month, which indicated that our new methodology was still not adequate for routine clinical application. Based on our experience with IAH usage, we recommend that mediumsized IAHs with an external diameter of the common iliac artery $<10 \mathrm{~mm}$ (i.e., small-sized IAH) should not be used for MHV reconstruction. If the external diameter of the common iliac artery is $10-12 \mathrm{~mm}$ (i.e., medium-sized IAH), the abovementioned surgical techniques can be applied depending on the branching shape of the IAH and the size and location of the graft V5/V8 openings. If the external diameters of the common and external iliac arteries are 15 and $10 \mathrm{~mm}$ respectively (i.e., large-sized IAH), the patency outcome would be comparable to that of iliac vein homografts $[2,6]$. Considering that a majority of IAHs are classified as small- or mediumsized, they should be very carefully selected to choose IAHs as a conduit material for MHV reconstruction.

We have preferentially performed MHV conduit anastomosis to the MHV-LHV stump as a separate method because it is technically simple and intuitive and it readily enables transjugular MHV stenting [12,13]. However, if the recipient MHV-LHV stump is small and/or a MHV conduit is small-sized, significant anastomotic stenosis can develop at this site. In such cases, unification of the MHV conduit and graft right hepatic vein orifice can be an option for secure outflow reconstruction of a modified right liver graft. To secure MHV conduit outflow however, the orifice of the MHV conduit should be sufficiently enlarged through patch plasty in combination with a longitudinal slit-like incision.

Our present study had several limitations of note. First, it was a single-center study with potential inherent selection bias in terms of the patients and IAHs. Second, it was a retrospective study involving a limited number of patients. Third, the designs for MHV reconstruction using IAHs were made on case-by-case 
basis and were thus not standardized.

In conclusion, our refined techniques for MHV reconstruction using IAHs have demonstrated improved short-term patency of the MHV conduit. However, they could not effectively prevent early conduit occlusion especially when small- or medium-sized IAHs were used. Individualized reconstruction designs are therefore necessary to achieve an effective MHV reconstruction using IAHs during LDLT using a modified right liver graft.

\section{ACKNOWLEDGEMENTS}

\section{Conflict of Interest}

No potential conflict of interest relevant to this article was reported.

\section{ORCID iD}

Gil-Chun Park: https://orcid.org/0000-0003-1631-3258

Shin Hwang: https://orcid.org/0000-0002-9045-2531

Dong-Hwan Jung: https://orcid.org/0000-0001-5984-023X
Tae-Yong Ha: https://orcid.org/0000-0001-9932-0212

Gi-Won Song: https://orcid.org/0000-0002-4235-0434

Chul-Soo Ahn: https://orcid.org/0000-0002-3844-3646

Deok-Bog Moon: https://orcid.org/0000-0002-8209-3540

Ki-Hun Kim: https://orcid.org/0000-0002-4016-0995

Young-In Yoon: https://orcid.org/0000-0002-9308-0366

Hwui-Dong Cho: https://orcid.org/0000-0001-8501-3385

Jin-Uk Choi: https://orcid.org/0000-0001-8078-0593

Sung-Gyu Lee: https://orcid.org/0000-0001-9161-3491

\section{Author Contribution}

Conceptualization: $\mathrm{SH}$

Formal Analysis: GCP, DHJ, GWS

Investigation: TYH, DBM, KHK

Methodology: SH, CSA, YIY, HDC, JUC

Project Administration: SH, SGL

Writing - Original Draft: SH, GCP

Writing - Review \& Editing: SH, DHJ, TYH, GWS, CSA, DBM, KHK, YIY, HDC, JUC, SGL

\section{REFERENCES}

1. Hwang S, Lee SG, Lee YJ, Sung KB, Park $\mathrm{KM}$, Kim KH, et al. Lessons learned from 1,000 living donor liver transplantations in a single center: how to make living donations safe. Liver Transpl 2006;12:9207.

2. Hwang S, Lee SG, Ahn CS, Park KM, Kim $\mathrm{KH}$, Moon DB, et al. Cryopreserved iliac artery is indispensable interposition graft material for middle hepatic vein reconstruction of right liver grafts. Liver Transpl 2005;11:644-9.

3. Sugawara Y, Makuuchi M, Akamatsu N, Kishi Y, Niiya T, Kaneko J, et al. Refinement of venous reconstruction using cryopreserved veins in right liver grafts. Liver Transpl 2004;10:541-7.

4. Hwang S, Lee SG, Song GW, Lee HJ, Park JI, Ryu JH. Use of endarterectomized atherosclerotic artery allograft for hepatic vein reconstruction of living donor right lobe graft. Liver Transpl 2007;13:306-8.

5. Hwang S, Jung DH, Ha TY, Ahn CS, Moon $\mathrm{DB}$, Kim KH, et al. Usability of ringed polytetrafluoroethylene grafts for middle hepatic vein reconstruction during living donor liver transplantation. Liver Transpl 2012;18:955-65.

6. Kim SH, Hwang S, Ha TY, Song GW, Jung DH, Ahn CS, et al. Usability of cryopreserved aortic allografts for middle hepatic vein reconstruction during livingdonor liver transplantation. J Gastrointest Surg 2016:20:1049-55.

7. Park GC, Hwang S, Ha TY, Song GW, Jung DH, Ahn CS, et al. Hemashield vascular graft is a preferable prosthetic graft for middle hepatic vein reconstruction in living donor liver transplantation. Ann Transplant 2019;24:639-46.

8. Ha TY, Hwang S, Jung DH, Ahn CS, Kim $\mathrm{KH}$, Moon DB, et al. Complications analysis of polytetrafluoroethylene grafts used for middle hepatic vein reconstruction in living-donor liver transplantation. Transplant Proc 2014;46:845-9.

9. Hsu SC, Thorat A, Yang HR, Poon KS, Li PC, Yeh CC, et al. Assessing the safety of expanded polytetrafluoroethylene synthetic grafts in living donor liver transplantation: graft migration into hollow viscous organs. Diagnosis and treatment options. Med Sci Monit 2017;23:3284-92.

10. Koc C, Akbulut S, Ozdemir F, Kose A, Isik B, Yologlu S, et al. Analysis of risk factors affecting the development of infection in artificial vascular grafts used for reconstruction of middle hepatic vein tributaries in living donor liver transplantation. Transplantation 2019; 103:1871-6.

11. Chung MH, Chuang CC, Liaw LF, Chen CY, Chen IM, Hsu CP, et al. Thrombotic ringed polytetrafluoroethylene graft with infection after living-donor liver transplantation. Transplant Proc 2018;50: 2606-10.

12. Ko GY, Sung KB, Yoon HK, Kim JH, Song HY, Seo TS, et al. Endovascular treatment of hepatic venous outflow obstruction after living-donor liver transplantation. J Vasc Interv Radiol 2002;13:591-9.

13. Ko GY, Sung KB, Yoon HK, Kim KR, Kim JH, Gwon DI, et al. Early posttransplant hepatic venous outflow obstruction: longterm efficacy of primary stent placement. Liver Transpl 2008;14:1505-11.

14. Kirchner VA, Hwang S, Song GW, Ahn 
CS, Moon DB, Kim KH, et al. Resolution of hepatic venous congestion following gradual occlusion of middle hepatic vein interposition graft in living donor liver transplantation. Ann Transplant 2016:21:619-25.

15. Hwang S, Lee SG, Park KM, Kim KH, Ahn CS, Lee YJ, et al. Hepatic venous conges- tion in living donor liver transplantation: preoperative quantitative prediction and follow-up using computed tomography. Liver Transpl 2004:10:763-70.

16. Ha TY, Kim YH, Chang JW, Park Y, Han Y, Kwon H, et al. Clinical outcomes of cryopreserved arterial allograft used as a vascular conduit for hemodialysis. J
Korean Med Sci 2016:31:1266-72.

17. Kwon H, Kwon H, Hong JP, Han Y, Park $\mathrm{H}$, Song GW, et al. Use of cryopreserved cadaveric arterial allograft as a vascular conduit for peripheral arterial graft infection. Ann Surg Treat Res 2015;89:514. 